\title{
Alert system for the VLBI database of ICRF2 sources. Preparatory case study.
}

\section{Elizaveta Rastorgueva-Foi ${ }^{* \dagger}$}

Aalto University Metsähovi Radio Observatory, Kylmälä, Finland

E-mail: elizaveta.rastorgueva@aalto.fi

\section{Nataliya Zubko}

Finnish Geodetic Institute, Masala, Finland

E-mail: nataliya.zubko@fgi.fi

\begin{abstract}
We discuss the role that extended structure of the ICRF2 defining sources may play in the accuracy of the determination of geodetic parameters. At the moment, there is no adequate way to take it into account on practice. We suggest a massive study of multifrequency behavior of the ICRF2 defining sources in order to determine signs of an approaching state of activity. In the near future this study will help to optimize the GeoVLBI scheduling process. We present a preliminary case study of a source $0133+476$. We found that the accuracy of the EOP determination is likely to be influenced by variability in the inner jet of the source rather than the large-scale extended emission.
\end{abstract}

11th European VLBI Network Symposium \& Users Meeting,

October 9-12, 2012

Bordeaux, France

\footnotetext{
* Speaker.

${ }^{\dagger}$ A footnote may follow.
} 


\section{Introduction}

The International Celestial Reference Frame (ICRF) is the radio-frequency realization of the International Celestial Reference System (ICRS). The ICRF is based on Very Long Baseline Interferometry (VLBI) observations of compact radio sources, most of which are Active Galactic Nuclei (AGN). These geodetic VLBI (GeoVLBI) observations are carried out at radio frequencies of 2 and $8 \mathrm{GHz}$, where $8 \mathrm{GHz}$ data is used for the actual ICRF parameter calculations, and $2 \mathrm{GHz}$ observations are used for ionospheric correction. The products of GeoVLBI are celestial coordinates of the reference sources, terrestrial coordinates of the observing antennas, and Earth Orientation $\mathrm{Pa}$ rameters (EOP) that link terrestrial and celestial reference frames. The second realization of ICRF (named ICRF2) is defined by the positions of 295 defining sources, and includes 3414 fiducial sources all together [1].

Astrometric and geodetic applications require astrometric and positional stability of defining sources. Also, due to the short length of scans in GeoVLBI observations, and an increasing number of relatively small $(\approx 12 \mathrm{~m}$ ) antennas used for these observations, relatively high brightness is the second important constraint. However, bright AGN sources tend to have a large amount of variable extended emission in a form of relativistic jet. They are highly variable also in total flux as well. Astrometric quality of the source is determined by VLBI delays deterioration due to the source structure. Fey and Charlot 1997 [2] suggested a criterion called structure index that determines astrometric suitability of the source. Due to variability of AGN, ICRF sources must be imaged frequently for determining their current structure indices. Hence, choice of the defining sources, based on the astrometric stability, favors the most compact objects [2]-5]. True proper motion of AGN are negligible due to their distance, however, variable AGN jets are able to mimic it for some sources. It should be noted also that not all apparent kinematic trends are correlated with the source structure [6-8]. There is an evidence that spectral difference in the source structure may also introduce an additional uncertainty to the calculated geodetic parameters via incorrect ionospheric correction [9]. To summarize, the source structure is one of the main limiting factors for the determination of ICRF, and it would be beneficial to take it into account in the GeoVLBI scheduling process.

At the moment structural variability of ICRF2 sources is normally detected post factum, after the GeoVLBI observations. Thus, the observational data that included the sources in question have been used, for example, by EOP services. Since ICRF coordinates of the fiducial sources are treated as global parameters for the calculation of EOP, the positional instability and possible apparent proper motion may influence their determination as well. Calibration and imaging of the GeoVLBI data is a time- and labor-consuming project, so the source structure may change on a faster time scale than it is monitored. In this way geodetic parameters, calculated with the use of extended source may be corrupted before any considerable structure changes are detected.

However, the current physical and kinematic models of AGN is built on multifrequency observations of AGN, including VLBI (e.g., [10]). In particular, the connection was found between emergence of the total flux flares (so-called active state) and ejection of the bright moving jet components (e.g., [11, 12]). Even though there is no satisfactory model that can accurately describe the apparent correlation between these two phenomena and predict behavior of any AGN, short-term regularities can be deduced for individual sources. 

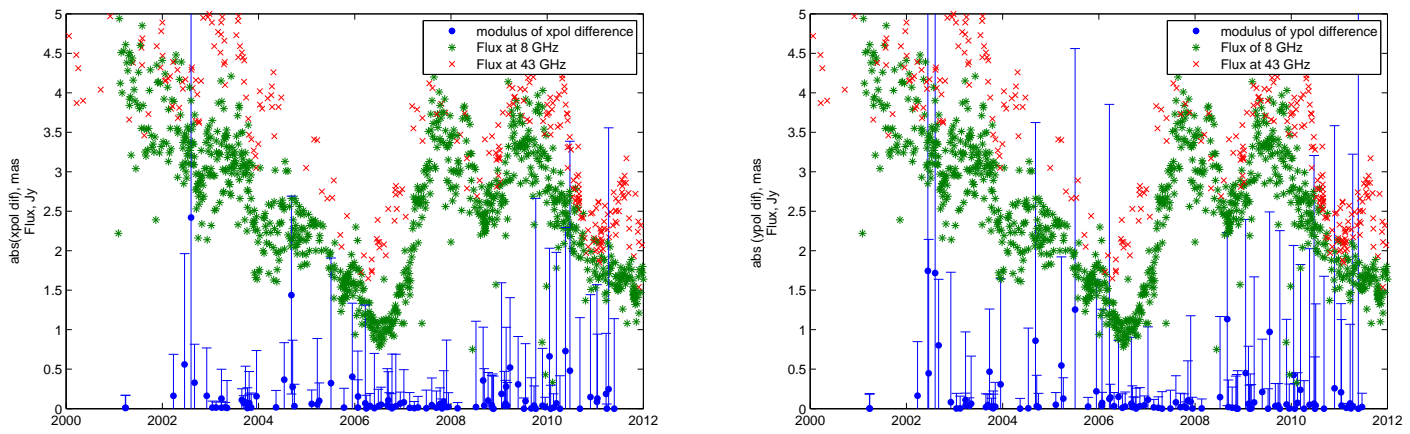

Figure 1: Modulus of differences of $x$ and $y$ polar coordinates, obtained including and excluding $0133+476$ from the analysis. Nutation shows similar pattern. Total flux light curves at 8 and $15 \mathrm{GHz}$ is superimposed on the image to mark active periods of the source.

For many ICRF2 sources astrophysical studies provided a vast amount of historical total flux monitoring data and long series of high-resolution VLBI images. These data can be used to deduce relations between the total flux variations at $\mathrm{mm}$ and $\mathrm{cm}$ wavelengths and VLBI components ejections for each individual ICRF2 defining sources. Upcoming monitoring data can be used to forecast, or, in worst cases, to make an educated guess of the beginning and duration of each source's active periods. These predictions may be sent as an alert to the GeoVLBI schedulers in order to encourage them to pay more attention at the "suspicious" sources or exclude them from the schedules for the whole active period.

Here we present first results of the preparatory work for this project. This is a case study of ICRF2 source $0133+476$, a quasar at $\mathrm{z}=0.859$ [13]. It has a one-sided jet propagating for up to 3 mas to the positional angle of $\approx 40^{\circ}$. This source was chosen randomly from those ICRF 2 defining sources that demonstrate prominent milliarcsecond-scale jet.

\section{Data reduction}

In this work we consider possible connection between variations in EOP, caused by the exclusion of an extended source from the process of data reduction, and structure variability of this source. We use total flux flares as a diagnostic of the source activity.

\subsection{EOP}

The analysis of GeoVLBI data has been performed with the least-squares method, where EOP were estimated using the software package VieVS [14]. For the analysis we selected only sessions, where the source $0133+476$ was observed during the time period 2001-2011. The total number of sessions that have been analyzed is 110 . First, the selected sessions were analyzed with the usual routine and EOP were estimated. In the second run the same sessions were used to estimate EOP, however, the observations of source $0133+476$ were excluded from the data. Then the difference between estimated solutions in these two runs was calculated for each session. Fig. 1 shows the modulus of differences obtained for $\mathrm{x}$ and $\mathrm{y}$ polar coordinates. 


\subsection{VLBI imaging}

For this analysis we used $15 \mathrm{GHz}$ VLBA self-calibrated visibility data for $0133+476$ from the MOJAVE survey [15]. Data reduction details and original images can be found in Lister at al. 2009 [16]. Kinematic results by MOJAVE team are presented in Lister at al. 2009 [17]. We have performed our own model-fitting with Gaussian circular and elliptical components using Difmap VLBI data reduction package [18] in order to quantify the emission of the jet. We tried to minimize $\chi^{2}$ and residual map rms for each epochs with a minimal number of components.

\subsection{Total flux radio observations}

The $8 \mathrm{GHz}$ source flux data was taken from online IVS database [20]. The $37 \mathrm{GHz}$ data were observed at Metsähovi Radio Observatory 14-m radio telescope. Details of observation and data reduction procedures could be found in Teräsranta 1998 [19]. Total flux light curves at 8 and $37 \mathrm{GHz}$ are superimposed on the EOP plot on Fig. 1.

\section{Results and discussion}

Clearly, that with the exclusion of a source from the GeoVLBI observation data we change the observation network. These changes must affect the estimated parameters regardless of the source structure. Though, our purpose is to reveal the possible effect of the specific source on the estimated parameters due to source structure and flux variability, thus, we try to evaluate possible connection of the parameter changes with the flux and structure variability.

Fig. 11 shows the modulus of differences obtained for $\mathrm{x}$ and $\mathrm{y}$ polar coordinates. There are few time intervals at around years 2002, 2005, and 2009-2011, when the accuracy of parameter estimation, which can be characterized by standard deviations, is getting worse. Similar trend is seen also in the nutation parameters, that we do not present here. We add light curves at 8 and $37 \mathrm{GHz}$ to the same figure to evaluate possible relation to the total flux variability. Visual inspection gives a hint that two main flares (years 2002 and 2009) and one sub-flare (year 2004) may precede periods of EOP "disturbance". Thus, it is possible to assume, that the difference of the estimated parameters and its accuracy may reveal an influence of source flux and structure variability.

To investigate connection to the source structure, we have scrutinized a series of VLBA images at $15 \mathrm{GHz}$. First, we would like to address an issue of legitimacy of using this frequency. VLBA resolution at $15 \mathrm{GHz}$ is comparable to that of IVS-R1 and R4 GeoVLBI sessions $(0.5-0.7$ mas depending on the longest baseline). Quasar 0133+476 is reported to have a spectral core shift between 8 and $15 \mathrm{GHz}$ of 0.099 mas [21], which is comparable to the uncertainties of the ICRF2 sources coordinate determination. This core shift is due to the different position of the optically thick component in the inner jet. In assumption of the outwards jet motion, this difference may lead to a time delay in the $8 \mathrm{GHz}$ VLBI structure changes with respect to the $15 \mathrm{GHz}$ structure. Similarity of the apparent jet structure and fluxes at these two frequencies suggests that it is unlikely that the time scale and magnitude of underlying kinematics of the jet, causing variability at these two frequencies, are fundamentally different at these two frequencies. We compare $15 \mathrm{GHz}$ VLBI data between different epochs in order to to investigate characteristic patterns of the structure changes. Next step in our analysis will be the same study performed for imaged $8 \mathrm{GHz}$ GeoVLBI data. 

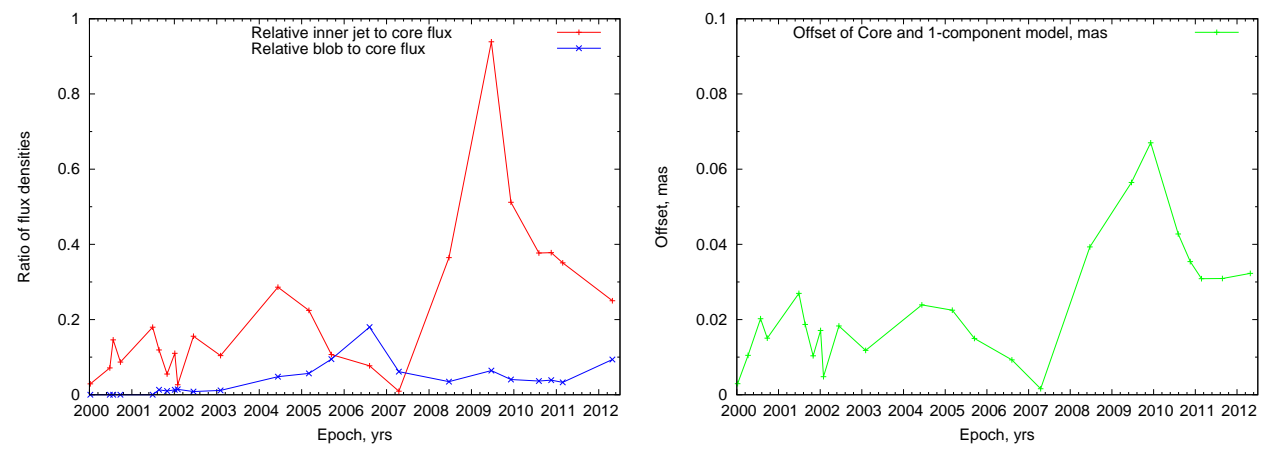

Figure 2: (Left panel) Ratio of the inner jet and the "blob" fluxes to the VLBI core flux. (Right panel) Offset of the one-Gaussian model of the extended source from the multi-Gaussian model core of the same source.

VLBA images and Gaussian models of 0133+476 show rather stable structure with the VLBI core, several neighboring components in the inner jet, located within 1 mas from the core, and a stationary extended "blob" at the distance of $\approx 2.7$ mas from the core. Faint extended "blobs" in the jets of quasars are likely to be kinematically and physically different from the brighter diffuse emission near the core. Thus, it seems natural to consider these regions of the jet separately. We plotted total fluxes of the inner jet and the "blob" as sum of Gaussian component fluxes, normalized to the core flux (as a flux of the core Gaussian component) on Fig. 2, left panel. This plot demonstrates that inner jet emission becomes dominant around epoch 2008.5. The time period of inner jet domination coincides with the most prominent EOP "disturbance" period. In contrast, brightening of the "blob" at roughly epoch 2006.5 can not be associated with any irregularities in EOP behaviour. This suggests that the accuracy of the EOP determination is more likely to be connected to the changes of brightness of the inner jet of a defining source, but not to the more extended, fainter jet component. We would like to qualitatively estimate contribution of the inner jet brightening on the accuracy of determination of an arbitrary source characterizing coordinate parameter, that is calculated under assumption that the source is compact. For that, we made a position uncertainty estimation in the following way. First, we model-fitted the source with only one elliptical Gaussian component. Size and shape of the component are taken from the IVS flux catalog. During Difmap model-fitting, both flux and position of the Gaussian source are allowed to vary in a wide range of values. Fig. \& right panel, presents the offset of the converged one-Gaussiancomponent model from the core of the multi-Gaussian-component model. The absolute value of the offset is small compare to the relative distances between components, and does not carry much information. However, its change suggests that the worsening of the position estimation accuracy follows the flux variations of the inner jet.

\section{Summary}

We found that:

- accuracy of EOP determination shows a sign of correspondence with the activity of a fiducial source: the accuracy is lower during the active state of the source;

- structure of inner jet (within one or two synthesized beam widths from the core) plays in this relationship bigger role than more extended jet structure. 


\section{Acknowledgments}

This research has made use of data from the MOJAVE database that is maintained by the MOJAVE team (Lister et al., 2009, AJ, 137, 3718). Authors would like to thank Brian Corey, Oleg Titov and Kaj Wiik for helpful discussions. Elizaveta Rastorgueva-Foi would like to thank RadioNet3 for the financial support for attending the conference.

\section{References}

[1] C. Ma et al., The Second Realization of the International Celestial Reference Frame by Very Long Baseline Interferometry, IERS Technical Note No. 35, 2009.

[2] A.L. Fey \& P. Charlot, ApJS 97 (111) 95

[3] A.L. Fey \& P. Charlot, ApJS 00 (128) 17

[4] P. Charlot, Source structure: an essential piece of information for generating the next ICRF, in Proceedings of the IAU Symp. No 248, 2007

[5] P. Charlot, Astrophysical Stability of Radio Sources and Implication for the Realization of the Next ICRF, in Proceedings of the Fifth IVS, 2008

[6] O. Titov, Proper motions of reference radio sources, in Proceedings of the Journes Systemes de Reference Spatio-temporels, 2007

[7] O. Titov, Systematic Effects in Apparent Proper Motions of Radio Sources, in Proceedings of the Fifth IVS, 2008

[8] A. Moor et al., AJ 11141

[9] S. Shabala et al., Quasar Structure Effects on the VLBI Reference Frame, Contribution to 7th IVS General Meeting, 2012

[10] A. Sokolov, A.P. Marscher, \& I.M. McHardy, AJ 04613 (725)

[11] S. Jorstad, AJ 10715 (362)

[12] U. Bach et al. $A \&$ \& 06456 (105)

[13] W.A. Barkhouse \& P.B. Hall AJ 01121 (2843)

[14] J. Böhm et al. The new Vienna VLBI Software VieVS, in Proceedings of the IAG Scientific Assembly, 2009

[15] http://www.physics.purdue.edu/astro/MOJAVE/sourcepages/0133+476.shtml

[16] M.L. Lister et al., AJ 09137 (3718)

[17] M.L. Lister et al., AJ 09138 (1874)

[18] M.C. Shepherd, M. C. in Astronomical Data Analysis Software and Systems VI, ASP, San Francisco, 1997

[19] H. Teräsranta et al. A\& AS 98 (132) 305

[20] http://lupus.gsfc.nasa.gov/sess/sesshtml/cumulative/source-perf-cumulative.html2

[21] A.B. Pushkarev et al., $A \&$ \& 12545 (A113) 